Check for updates

Cite this: RSC Adv., 2019, 9, 26588

Received 12th June 2019

Accepted 13th August 2019

DOI: $10.1039 / c 9 r a 04414 k$

rsc.li/rsc-advances

\title{
Sulfur-doped graphene quantum dot-based paper sensor for highly sensitive and selective detection of 4-nitrophenol in contaminated water and wastewater $\dagger$
}

\begin{abstract}
Nguyen Thi Ngoc Anh, ${ }^{a}$ Pei-Yi Chang ${ }^{b}$ and Ruey-An Doong (DD *ac
4-Nitrophenol (4-NP) is a promulgated priority pollutant, which can cause a negative impact on human health. The development of a direct and effective technique for the rapid detection and screening of 4NP is, therefore, of urgent need. In this study, the blue luminescent sulfur-doped graphene quantum dots (S-GQDs) with a size of 1-5 nm are fabricated using a one-step pyrolysis procedure in the presence of citric acid and 3-mercaptosuccinic acid. The S-GQDs exhibit a strong emission band at $450 \mathrm{~nm}$ under the excitation of $330 \mathrm{~nm}$ UV light. 4-NP can serve as the fluorescence quencher by the $\pi-\pi$ interaction with S-GQD, resulting in the linear decrease in fluorescence intensity after the addition of various 4-NP concentrations ranging from $10 \mathrm{nM}$ to $200 \mu \mathrm{M}$. The S-GQDs serve as the sensing probe to enhance the analytical performance on 4-NP detection with the limit of detection values of 0.7 and $3.5 \mathrm{nM}$ in deionized water and wastewater, respectively. The S-GQD based sensing platform can be used to detect 4-NP in different matrices of water and wastewater. In addition, the detected percentages of spiked 4NP concentrations in the presence of different matrices and interferences are in the range of $(98 \pm 5)-$ $(108 \pm 2) \%$. Moreover, the S-GQD based paper sensor can rapidly screen 4-NP in wastewater within $1 \mathrm{~min}$. Results obtained in this study clearly demonstrate the superiority of S-GQDs as a promising fluorescence probe for highly sensitive and selective detection of a wide concentration range of 4-NP in deionized water and wastewater.
\end{abstract}

\section{Introduction}

Nitrophenols such as 4-nitrophenol (4-NP) and 2,4-dinitrophenol are among the most recalcitrant organic pollutants in soil and aquatic environments, and can cause serious environmental problems due to their chemical stability and poor biodegradability. ${ }^{1}$ 4-NP is one of the priority pollutants promulgated by the United States Environmental Protection Agency and the lifetime health advisory level is reported to be 60 $\mu \mathrm{g} \mathrm{L}^{1}{ }^{2}$ Although several Au-based nanomaterials such as $\mathrm{Au}-$ $\mathrm{Fe}_{3} \mathrm{O}_{4}$ nanostructures ${ }^{3,4}$ and $\mathrm{Pt}-\mathrm{Au} @$ oreduced graphene oxide $(\mathrm{rGO})^{5}$ have been developed to decompose 4-NP in aquatic environments, a simple and low-cost analytical method for the

\footnotetext{
anstitute of Environmental Engineering, National Chiao Tung University, 1001, University Road, Hsinchu 30010, Taiwan

${ }^{b}$ Center for Measurement Standard, Industrial Technology Research Institute (ITRI), 321, Sec. 2, Kuang Fu Road, Hsinchu, 30011, Taiwan

${ }^{c}$ Department of Biomedical Engineering and Environmental Sciences, National Tsing Hua University, 101 Section 2, Kuang-Fu Road, Hsinchu, 30013, Taiwan. E-mail: radoong@mx.nthu.edu.tw
}

$\dagger$ Electronic supplementary information (ESI) available. See DOI: $10.1039 / \mathrm{c} 9 \mathrm{ra} 04414 \mathrm{k}$ rapid and effective monitoring of residual 4-NP in water and wastewater is still of urgent need.

The traditional technique for the determination of 4-NP include gas chromatography, capillary electrophoresis, high performance liquid chromatography and electrochemical method. ${ }^{6-9}$ However, these methods usually need expensive instruments and tedious procedures for analysis. More recently, fluorescence spectrophotometry has been developed for highly sensitive detection of 4-NP. ${ }^{10-12}$ Yang et al. ${ }^{10}$ have used BSA Aunanocrystals as a fluorescence probe to detect 4-NP in deionized water. Several nanomaterials including molecularly imprinting polymer-carbon dots (MIP-CD) and CdTe have also been synthesized as the fluorescence probe for 4-NP detection in deionized water. ${ }^{11,12}$ However, the sensitivity of these MIPbased sensing probes is not high and the limit of detection (LOD) are usually in the range of 40-60 nM. In addition, only deionized or river water was used as the water matrix and the information on the application of sensing probe to detect 4-NP in the complicated matrix is limited.

Graphene quantum dots (GQDs) is a newly developed fluorescence materials with excellent photoluminescence, good biocompatibility, low toxicity, easy fabrication, and large surface area.$^{13-19}$ GQDs have recently been used as the sensing 
materials for the detection of a wide variety of analytes including metal ions, organics, and biomolecules. ${ }^{\mathbf{2 0 - 2 4}}$ Usually the sensitivity of carbon-based quantum dots including carbon dots (CD) and GQDs toward analyte detection is dependent on quantum yield, which is related to the layers of quantum dots and dopants. The layer of GQDs is, in general, $<10$ layers and has less influence on the quantum yield in comparison with CD. Therefore, doping of graphic carbons with heteroatoms such as $\mathrm{P}, \mathrm{N}, \mathrm{B}$ and $\mathrm{S}$ atoms can not only amplify the photoluminescence behavior but also increase the quantum yield, resulting in the enhancement of sensing sensitivity and selectivity. ${ }^{25-29}$ In particular, doping with sulfur atom is relatively difficult in comparison with other dopants because of its electronegativity. ${ }^{30}$ Up to now, only limited studies on the fabrication of S-doped GQDs (S-GQDs) have been reported for sensing applications. ${ }^{26,31-33} \mathrm{Li}$ et al. ${ }^{31}$ have used the top-down electrolysis method to fabricate S-GQDs from graphite for Fe ion detection. However, the particle size distribution of S-GQD is wide and inhomogeneous. Several studies have used 1,3,6-trinitropyrene and different sulfur-containing chemicals as the carbon and sulfur sources, respectively, for the fabrication of S-GQD to detect metal ions including $\mathrm{Pb}^{2+}$ and $\mathrm{Ag}^{+}{ }^{32,33} \mathrm{Up}$ to now, the development of S-GQD based sensing element for the detection of organic chemicals has received less attention.

Our previous work has synthesized Au@S-GQD nanocomposites for the detection of 4-NP using UV-visible absorption spectra. The ratiometric detection based on the change in wavelength ratio between 307 and $530 \mathrm{~nm}$ from 4-aminophenol, the reduction product of $4-\mathrm{NP}$, and surface plasma resonance of $\mathrm{Au}$ nanoparticles, respectively, was used to detect 4-NP. ${ }^{34}$ Although low limit of detection (LOD) in deionized water was obtained, the analytical performance is highly dependent on the size of Au nanoparticle and the reduction efficiency of 4-NP. Therefore, a simple and straightforward method for the rapid and effective detection of 4-NP may thus be needed. However, the use of pure S-GQDs as the fluorescence probe for directly detection of 4-NP has not been yet reported. Moreover, the analytical performance of S-GQD fluorophore toward 4-NP detection in different water and wastewater matrices remains unclear.

Herein, we have developed a simple and cost-effective onestep pyrolysis method for the fabrication of S-GQDs using citric acid and 3-mercaptosuccinic acid as the carbon and sulfur sources, respectively. The as-prepared S-GQDs are then used as the fluorescence probe to effectively detect 4-NP in aqueous solution with complex matrix. Scheme 1 illustrates the possible reaction mechanism of S-GQDs for the detection of 4-NP. The as-synthesized S-GQDs exhibit a distinct fluorescence peak at $450 \mathrm{~nm}$ under the excitation of $330 \mathrm{~nm}$ UV light at pHs of 5-9, which is highly sensitive and selective toward 4-NP detection. To the best of our knowledge, this is the first report on using fluorescent S-GQDs as the sensing platform for 4-NP detection in a wide variety of wastewaters. Addition of 4-NP effectively quenches the fluorescent intensity by $\pi-\pi$ interaction and a dynamic range of 4 orders of magnitude with LOD of $0.7 \mathrm{nM}$ in deionized water and $3.5 \mathrm{nM}$ in wastewater is observed. Moreover, the S-GQD coated paper-based sensing technique has been

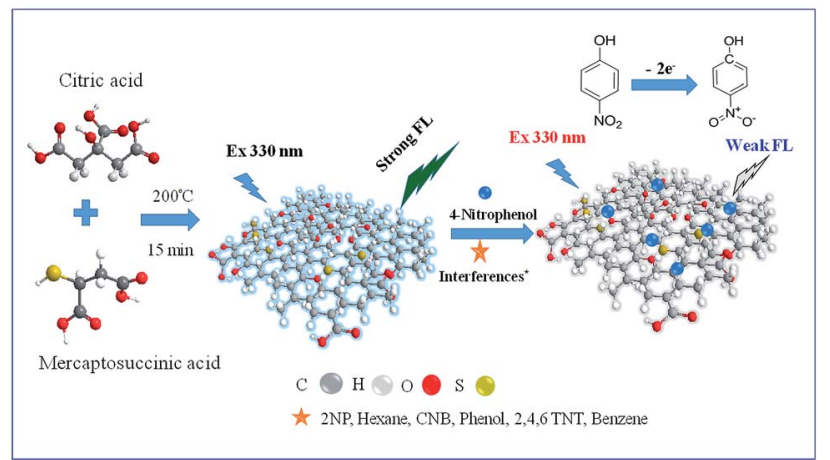

Scheme 1 Schematic illustration of the fluorescence sensing of 4nitrophenol by S-doped GQDs in real wastewater samples with different matrices.

developed to rapidly screen 4-NP in real wastewater samples within $1 \mathrm{~min}$.

\section{Experimental section}

\section{Fabrication of S-GQDs}

The S-GQDs was synthesized using one-step pyrolysis method according to our previous report with minor modification. ${ }^{34}$ Briefly, $2.0 \mathrm{~g}$ citric acid (CA) and $27-240 \mathrm{mg}$ of 3-mercaptosuccinic acid (MSA, Sigma-Aldrich) were added into $0.5 \mathrm{~mL}$ of ultrapure deionized water $(18.2 \mathrm{M} \Omega \mathrm{cm})$ to get the $\mathrm{MSA} / \mathrm{CA}$ ratio of $0.0135-0.12$. Then mixture was heated to $200{ }^{\circ} \mathrm{C}$ in an oven and maintained for $15 \mathrm{~min}$ to produce S-GQDs with strong fluorescence. The S-GQDs with the bright yellow suspension were dialyzed with $1 \mathrm{k}$ dialysis bag for $24 \mathrm{~h}$ by adding ultrapure deionized water regularly to remove the residual chemicals. The harvested S-GQDs in deionized water were stored at room temperature.

\section{Characterization}

The optical property including UV-visible and fluorescence spectra of as-synthesized S-GQDs was determined by Hitachi U4100 UV-VIS-NIR and F-7000 fluorescence spectrophotometers (Tokyo, Japan), respectively. The topography as well as thickness of S-GQDs was investigated using Agilent 5500 scanning probe microscope in tapping mode. The morphology and particle size distribution of S-GQDs were examined using a transmission electron microscope (TEM, JEOL, JEMARM200F, Tokyo, Japan) and a high-resolution TEM (HRTEM, JEOL JEM-2010) at $200 \mathrm{kV}$. X-ray photoelectron spectroscopy (XPS) was used to characterize the change in chemical species of S-GQDs by an ESCA Ulvac-PHI 1600 photoelectron spectrometer from Physical Electronics using $\mathrm{Al} \mathrm{K} \alpha$ radiation photon energy at $1486.6 \pm 0.2 \mathrm{eV}$. X-ray diffraction (XRD) patterns were recorded using a Bruker D8 X-ray diffractometer with Ni filtered $\mathrm{Cu} \mathrm{K} \alpha$ radiation $(\lambda=1.5406 \AA)$. Additionally, Raman spectra of S-GQDs were determined by using Bruker Senterra micro-Raman spectrometer equipped with an Olympus BX 51 microscope and an Andor DU420-OE CCD camera. The 
Fourier transform infrared (FTIR) spectra of S-GQDs were recorded with a Horiba FT720 spectrophotometer.

\section{Detection of 4-NP in deionized water by S-GQDs}

The detection of 4-NP by S-GQDs was performed at room temperature based on the fluorescence quenching principle. To understand the concentration effect of S-GQDs on 4-NP detection, different S-GQD concentrations at 0.19 and $0.4 \mathrm{mg} \mathrm{mL}^{-1}$ were tested. As illustrated in Fig. S1 (ESI $\dagger$ ), the change in fluorescence of S-GQDs at 0.19 and $0.4 \mathrm{mg} \mathrm{mL}^{-1}$ decreases from 1.0 \pm 0.02 to $0.14 \pm 0.01$ and $0.135 \pm 0.007$, respectively, when 50 $\mu \mathrm{M} 4$-NP were added. This result clearly indicates that the SGQD concentration at $0.19-0.4 \mathrm{mg} \mathrm{mL}^{-1}$ has little influence on the analytical performance of S-GQDs toward 4-NP detection, presumable attributed to the fact that S-GQDs can provide sufficient reaction sites for 4 -NP in the tested concentration range. Therefore, $10 \mu \mathrm{L}$ of $19 \mathrm{mg} \mathrm{mL}^{-1}$ S-GQDs was added into $1 \mathrm{~mL}$ of deionized water to get the final S-GQD concentration of $0.19 \mathrm{mg} \mathrm{mL} \mathrm{m}^{-1}$. Various volumes of 4-NP stock solution were added to the S-GQD solution to get the final concentration of $0.01-200 \mu \mathrm{M}$. A total volume of $2 \mathrm{~mL}$ was used to determine the analytical performance of S-GQDs. All the mixtures were incubated for $1 \mathrm{~min}$ at room temperature and then the fluorescence signal of all the mixtures was measured from 350 to $600 \mathrm{~nm}$ after the excitation of $330 \mathrm{~nm}$. The calibration curve of 4-NP was determined by the change in fluorescence intensity before and after the addition of different concentrations of target compound. To evaluate the $\mathrm{pH}$ effect, all the solutions were adjusted to pHs 5-9 by $0.1 \mathrm{M} \mathrm{HCl}$ or $\mathrm{NaOH}$ after the addition of $50 \mu \mathrm{M} 4$-NP. Moreover, the selectivity of sensing system was evaluated by adding other 9 nitroaromatic analogues including 2-nitrophenol (2-NP), 1-chloro-4-nitrobenzene (CNB), 2,4-,6tronitrotoluene (TNT), hexane, and aromatic compounds (benzene, phenol, catechol, hydroquinone, and resorcinol) as the interferences into S-GQD solution and then the quenching effect of fluorescence was investigated after $1 \mathrm{~min}$ of incubation. All the experiments were performed in at least triplicate to ensure the quality control. The precision of intra and inter batches were controlled within 5 and $3 \%$, respectively. In addition, the reproducibility of intra and inter samples during day studies was both controlled within $5 \%$.

\section{Detection of 4-NP in wastewater samples}

To evaluate the feasibility of using S-GQDs as the sensing probe to detect 4-NP in environmentally aqueous samples, standard addition method was used to spike various concentrations of 4NP into different matrices of water and wastewaters including lake water and 3 effluents from wastewater treatment plants. The lake water was sampled from Cheng-Kung Lake in the campus of National Tsing Hua University (Hsinchu, Taiwan), while effluents from wastewater treatment plants were collected from metal industry, sewage and electroplating factory in Taoyuan City, Taiwan. The physicochemical property of the water and wastewater is shown in Table S1 (ESI $\dagger$ ). To remove the suspended solids and impurities in wastewaters, all the solutions were first filtrated by $0.45 \mu \mathrm{m}$ Millipore filter paper followed by C-18 SPE column. ${ }^{24}$ After the filtration, various concentrations of 4-NP at $0.05-200 \mu \mathrm{M}$ were spiked into the real samples and then S-GQDs were added into the solutions. The fluorescence signal of the mixture was then measured immediately to evaluate the matrix effect of environmental samples on 4-NP detection by S-GQDs. Moreover, the traditional UVvisible method was used to determine the recovery of 4-NP in deionized water for comparison.

\section{Fabrication of S-GQDs-based paper sensors}

The S-GQDs based paper strip was fabricated according to our previously reported results. ${ }^{24}$ Briefly, the cellulose filter paper with diameter of $125 \mathrm{~mm}$ and pore size of $25 \mu \mathrm{m}$ was cut into 1 $\times 3.5 \mathrm{~cm}^{2}$ strips. The paper strip was spiked with $0.19 \mathrm{mg} \mathrm{mL}^{-1}$ S-GQDs solution for $10 \mathrm{~min}$ and then dried in an oven to form the paper-based sensing probe. The applicability of paper-based sensing probe was evaluated by immersing the paper strip into wastewater spiked with 0.1-500 $\mu \mathrm{M} 4$-NP for $1 \mathrm{~min}$. To achieve the visual detection on the change in fluorescence intensity, the paper based sensor was then irradiated with a $330 \mathrm{~nm}$ UV lamp and the optical image was recorded by a Sony digital camera. Moreover, the specificity of S-GQDs based paper sensor toward 4-NP detection was examined with other aromatic and nitroarene interferences.

\section{Results and discussion}

\section{Optimization of fabrication of S-GQDs}

The fluorescence property as well as analytical performance of S-GQDs is highly dependent on the fabrication conditions. Therefore, the mass ratio of MSA/CA and the $\mathrm{pH}$ value during the fabrication of S-GQDs were first optimized. Fig. S2 (ESI $\dagger$ ) shows the fluorescence intensity of S-GQDs prepared at various MSA/CA ratios. High fluorescence intensity of S-GQDs at MSA/ CA ratio of 0.02 is clearly observed in comparison with other $\mathrm{MSA} / \mathrm{CA}$ ratios, and, therefore, addition of $40 \mathrm{mg}$ MSA is further used for the preparation of S-GQDs. Moreover, the influence of solution $\mathrm{pH}$ at 2-7 on the fabrication of S-GQDs was further explored. Since the original $\mathrm{pH}$ value of citric acid is around 2, $\mathrm{NaOH}$ pellets were used to adjust the solution $\mathrm{pH}$ to 5 and 7 for the fabrication of S-GQDs at MSA/CA ratio of 0.02. As shown in Fig. S3 (ESI $\dagger$ ), the maximum fluorescence intensity of the asprepared S-GQDs decreases when $\mathrm{pH}$ increases from 2 to 7. The decrease in fluorescence intensity of S-GQDs upon the increase in solution $\mathrm{pH}$ is mainly attributed to the relationship between the solution $\mathrm{pH}$ and the $\mathrm{p} K_{\mathrm{a}}$ of citric acid. The $\mathrm{p} K_{\mathrm{a}}$ values of triprotic citric acid are 3.13, 4.76 and 6.40, respectively. This means that citric acid is in its molecular form at low solution $\mathrm{pH}$, which is conducive to the fabrication of S-GQD during the pyrolysis at $200{ }^{\circ} \mathrm{C}$. After addition of $50 \mu \mathrm{M} 4-\mathrm{NP}$, the fluorescence intensity of S-GQDs prepared at $\mathrm{pH} 2$ decreases obviously because of the initially high fluorescence intensity. However, the fluorescence intensity of S-GQDs prepared at $\mathrm{pH}$ 5-7 only changes slightly when high concentration of 4-NP at $200 \mu \mathrm{M}$ is added. This result clearly indicates that $\mathrm{pH}$ may 

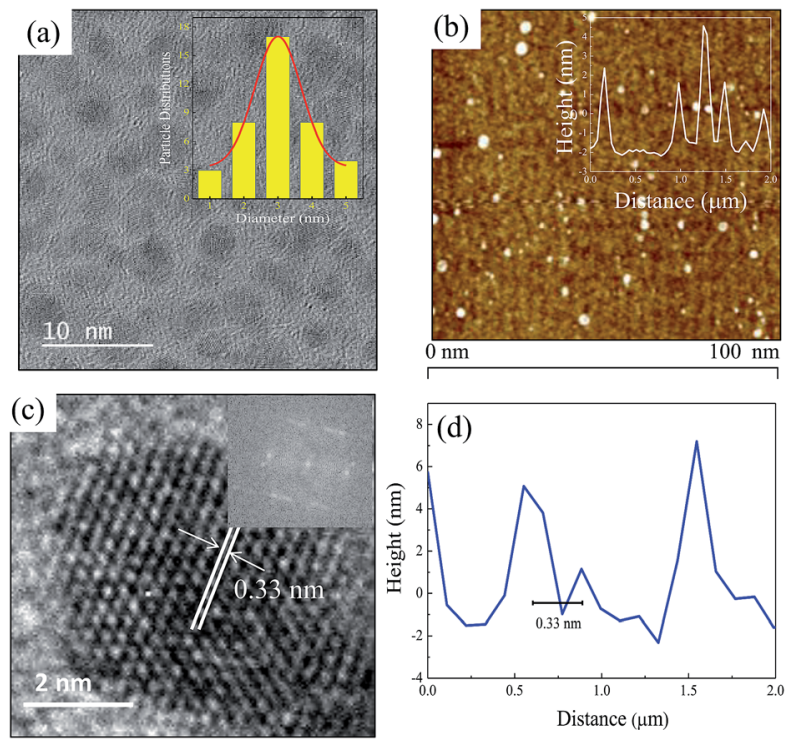

Fig. 1 The (a) TEM image and histogram (inset, $n=50$ ), (b) AFM image, roughness and height profile (inset), (c) HRTEM image and fast Fourier transform pattern (inset) of single S-GQDs, and (d) lattice spacing of as-prepared S-GQDs.

influence the analytical performance, and only S-GQDs prepared at $\mathrm{pH} 2$ show excellent ability toward 4-NP detection.

\section{The structural characterization of S-GQDs}

Fig. 1a shows the TEM image and histogram of the as-prepared S-GQDs. The as-prepared S-GQDs are quite homogeneous and uniformly distributed with particle and mean diameters of 1-5 and $3 \mathrm{~nm}$, respectively. The atomic force microscopic image illustrated in Fig. 1b indicates that height profile is in the range of 1.5-4.0 nm with an average size of $3.0 \mathrm{~nm}$, confirming the successful formation of S-GQDs with 3-8 layers in thickness (inset of Fig. 1b). Moreover, the surface roughness shown in the below of Fig. $1 \mathrm{~b}$ is relatively smooth and uniform, reflecting the successful fabrication of homogeneous S-GQDs. The HRTEM image displays the fringes of carbon lattice of S-GQDs (Fig. 1c). The fast Fourier transform (FFT) pattern also corroborates that the S-GQDs are single crystal (inset Fig. 1c). The lattice spacing in Fig. 1d deciphers the average inter-planar of $0.33 \mathrm{~nm}$, which corresponds to the (002) peak of graphitic carbon. The XRD pattern of S-GQDs (Fig. S4, ESI $\uparrow$ ) also exhibits a typically broad (002) plane of graphene peak at $24.3^{\circ} 2 \theta$, clearly indicating the successful fabrication of GQD nanosheets.

The optical property of as-prepared S-GQDs was explored by UV-visible and fluorescence spectra. As shown in Fig. 2A, the S-GQDs exhibit UV-visible spectra with an absorption peak at $280 \mathrm{~nm}$. Meanwhile, a shoulder peak at $230 \mathrm{~nm}$, which corresponds to the $\pi-\pi^{*}$ transition of $\mathrm{sp}^{2}$ domain, is also observed. The absorption band at $280 \mathrm{~nm}$ is attributed to the $\mathrm{n}-\pi^{*}$ transition of carbonyl groups. ${ }^{14}$ Moreover, the SGQDs exhibit good fluorescent property and a strong emission wavelength at $450 \mathrm{~nm}$ is obtained upon the excitation of $330 \mathrm{~nm}$ UV light. To further elucidate the fluorescence
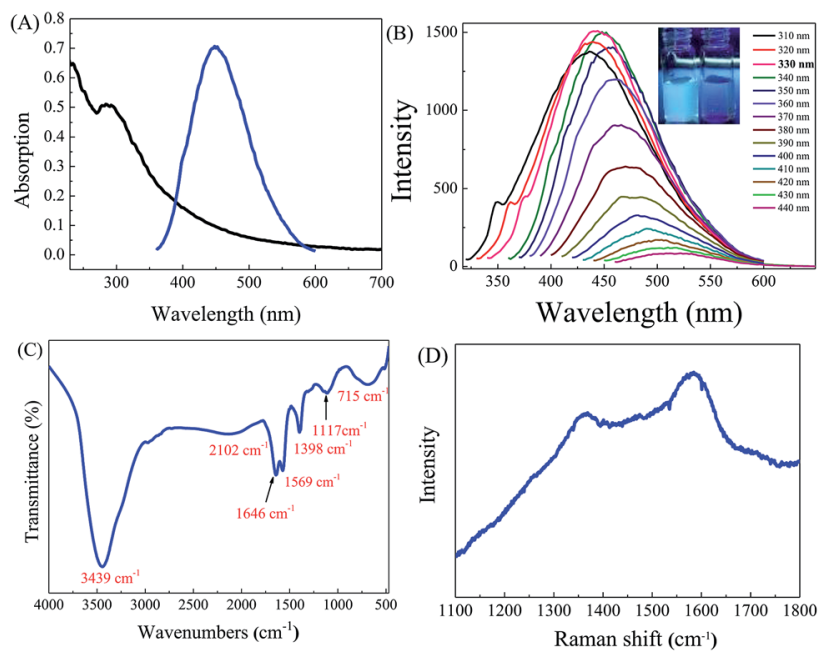

Fig. 2 The (A) UV-visible and fluorescence spectra, (B) fluorescence emission spectra under the irradiation of excitation wavelengths at $310-440 \mathrm{~nm},(\mathrm{C})$ the FTIR and (D) Raman spectra of the as-prepared SGQDs.

property of S-GQDs, the emission spectra of S-GQDs were recorded after the excitation of UV light at $310-440 \mathrm{~nm}$. As illustrated in Fig. 2B, the wavelength peak of S-GQDs redshifts from 450 to $530 \mathrm{~nm}$ with the increase in excitation wavelength. Besides, the emitted fluorescence intensity increases at short wavelength of $310-330 \mathrm{~nm}$ and then decreases upon increasing the wavelength from 340 to $440 \mathrm{~nm}$, which is consistent with the previous reports. ${ }^{15,23,24}$ These obtained optical properties clearly indicate that SGQDs can produce highest fluorescence intensity at $330 \mathrm{~nm}$, which may exhibit the superior photoluminescence property to detect 4 -NP.

The quantum yield of fluorophore plays an important role in fluorescent sensing system. In this study, the fluorescence quantum yield, determined by using fluorescein as the standard fluorophore, is calculated to be $11 \%$, which is satisfactory for sensing application. Dutta Chowdhury and Doong ${ }^{23}$ has used pyrolysis method to fabricate GQDs using citric acid as the carbon source and found that the quantum yield of assynthesized GQDs was $10.2 \%$. Several studies have also indicated that the quantum yield of GQD based materials can be enhanced by the introduction of heteroatoms including $\mathrm{N}, \mathrm{S}$, and B into GQDs..$^{24,31,35}$ The relatively high quantum yield of SGQDs may be possibly ascribed to the existence of $\mathrm{S}-\mathrm{OH}$ and $\mathrm{S}=\mathrm{O}$ on the surface of GQDs. The EDS spectrum of as-prepared S-GQDs from TEM image shows that the atomic percentage of $\mathrm{C}, \mathrm{O}$ and $\mathrm{S}$ is $89.7,6.0$ and $4.3 \mathrm{wt} \%$, respectively (Fig. S5, ESI $\dagger$ ), and the content of sulfur atom in GQDs ( $4.3 \mathrm{wt} \%)$ is higher than the previous reports. ${ }^{26,31}$ The doping of anions such as B and S into graphic carbon can serve as the electron trap center to change the electron density of graphic carbon, ${ }^{25,35}$ resulting in the improvement the electrical features as the electronic band structures are altered to a significant extent.

The FTIR and Raman spectra were further applied to examine the functional groups and carbon structures of as- 
prepared S-GQDs. Fig. 2C shows the FTIR spectrum of asprepared S-GQDs. The S-GQDs exhibit a strong O-H peak at $3439 \mathrm{~cm}^{-1}$, indicating the good hydrophilic property of S-GQDs. A small and broad peak at $2966 \mathrm{~cm}^{-1}$ is the stretching vibration of $\mathrm{C}-\mathrm{H}$ functional group in graphitic backbone of S-GQDs. Moreover, the small bands at $2102 \mathrm{~cm}^{-1}$ and $1569-1646 \mathrm{~cm}^{-1}$ are also from the bending vibration of $\mathrm{C}-\mathrm{C}$ and $\mathrm{C}=\mathrm{C}$ bonds of graphitic backbone, respectively. Several sulfur-containing functional groups from the stretching vibration of $\mathrm{S}=\mathrm{O}, \mathrm{C}=\mathrm{S}$ and $\mathrm{S}-\mathrm{OH}$ functional groups at 1398, 1117 and $715 \mathrm{~cm}^{-1}$, respectively, indicate the successful doping of $\mathrm{S}$ atoms onto carbon backbone of S-GQDs. ${ }^{36,37}$ Moreover, Raman spectrum is also provided in this study to characterize the carbon-based materials. As shown in Fig. 2D, two peaks located at 1360 and $1584 \mathrm{~cm}^{-1}$ are the characteristic peaks of $\mathrm{D}$ and $\mathrm{G}$ bands, respectively. The $I_{\mathrm{D}} / I_{\mathrm{G}}$ ratio of 0.94 implies the decrease in defect of $\mathrm{sp}^{2}$ carbon lattice. ${ }^{38}$

XPS was further used to identify the chemical species of elements in S-GQDs. The survey scan of XPS spectra shows O 1s, S 1s, S 2s and S 2p peaks at 532, 284, 168 and $162 \mathrm{eV}$, respectively (Fig. 3A). After peak deconvolution, the C 1s spectrum of S-GQDs (Fig. 3B) exhibits four peaks at 283.9, 284.5, 285.5 and $287.5 \mathrm{eV}$, which can be assigned as $\mathrm{C}-\mathrm{C}, \mathrm{C}=\mathrm{C}, \mathrm{C}-\mathrm{S}$ and $\mathrm{C}=\mathrm{O}$ functional groups, respectively. ${ }^{26,39}$ The deconvoluted $\mathrm{O}$ 1s spectrum shows the characteristic $\mathrm{C}-\mathrm{O}, \mathrm{C}=\mathrm{O}$, and $\mathrm{C}-\mathrm{OH} / \mathrm{C}-\mathrm{O}-\mathrm{C}$ peaks at 531.5, 531.9 and $533.5 \mathrm{eV}$, respectively (Fig. 3C).$^{39,40,41}$ Moreover, the S 2p signal of S-GQDs contains two peaks at 161.6 and $168.5 \mathrm{eV}$, respectively, which are the $S 2 p_{3 / 2}$ and $S 2 p_{1 / 2}$ peaks of spin-orbit coupling of $\mathrm{S}^{2-}$ or oxidized S species $\left(-\mathrm{SO}_{n}{ }^{-}\right)$(Fig. 3D). ${ }^{32,42}$ These results clearly indicate that $\mathrm{S}$ atoms are successfully doped, and can react with the abundant O-containing functional groups for the enhanced detection of 4-NP by S-GQDs.

\section{Detection of 4-NP by S-GQDs}

The fluorescence intensity of fluorophore is highly dependent on solution $\mathrm{pH}$ and, therefore, the effect of $\mathrm{pH}$ on the
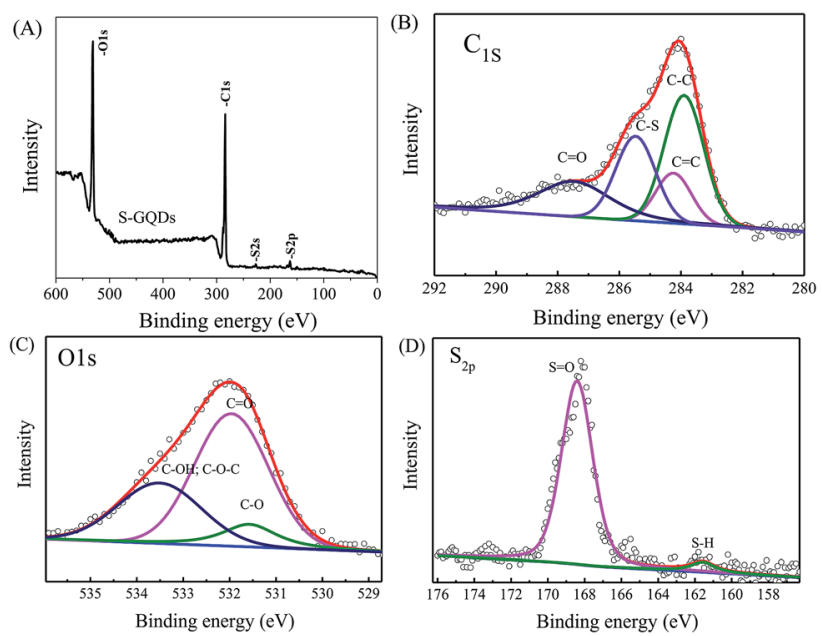

Fig. 3 XPS spectra of (A) survey spectra and deconvoluted (B) C 1s, (C) $\mathrm{O} 1 \mathrm{~s}$, and (D) S 2p peaks of as-prepared S-GQDs. fluorescence intensity of S-GQDs was evaluated. Fig. S6 (ESI†) shows the fluorescence intensity of S-GQDs as a function of $\mathrm{pH}$ ranging from 5 to 9 . The emitted fluorescence intensity of $\mathrm{S}$ GQDs increases under weakly acidic conditions ( $\mathrm{pH} \mathrm{5-7)} \mathrm{and}$ then reaches the plateau when $\mathrm{pH}$ is $>7$. After addition of 200 $\mu \mathrm{M} 4-\mathrm{NP}$, the fluorescence intensity decreases dramatically, indicating the good quenching effect of 4-NP on the fluorescence emission of S-GQDs. Fig. 4a shows the change in fluorescence intensity of S-GQDs as a function of $\mathrm{pH}$. The fluorescence intensity of S-GQDs increases rapidly from $\mathrm{pH} 5$ to 7 and then slowly reaches the maximum fluorescence intensity at $\mathrm{pH}$ 7-9. In addition, the response time of S-GQDs was investigated. As shown in Fig. 4b, the fluorescence intensity decreases dramatically within $1 \mathrm{~min}$ and remain constant after $5 \mathrm{~min}$, clearly indicating the rapid response of the developed sensor for the detection of 4-NP. Therefore, $\mathrm{pH} 7$ and reaction time of 1 min are selected in this study for further experiments.

The analytical performance of S-GQDs toward 4-NP detection was evaluated by adding 4-NP into the solution containing $0.19 \mathrm{mg} \mathrm{mL}^{-1} \mathrm{~S}$-GQDs. Fig. 5A shows the fluorescence spectra of S-GQDs in deionized water in the presence of 0.01-200 $\mu \mathrm{M} 4-\mathrm{NP}$. The fluorescence intensity at $450 \mathrm{~nm}$ decreases obviously upon the increase in 4-NP concentration. A 94\% decrease in fluorescence peak intensity is observed when 4-NP concentration increases from 0 to $200 \mu \mathrm{M}$. In addition, the peak wavelength shifts from 450 to $472 \mathrm{~nm}$, which is mainly attributed to the $\pi-\pi$ interaction and hydrogen bonding between 4-NP and S-GQDs. Although the maximum peak shifts slightly after the addition
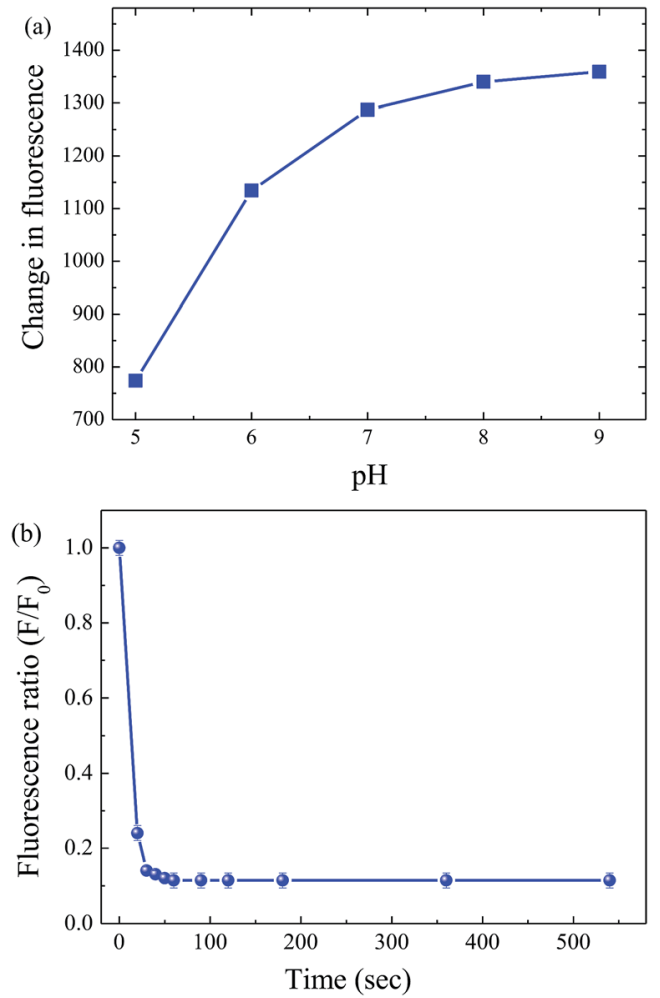

Fig. 4 The change in fluorescence intensity of S-GQDs as a function of (a) $\mathrm{pH}$ and (b) response time after the addition $200 \mu \mathrm{M} 4-\mathrm{NP}$. 

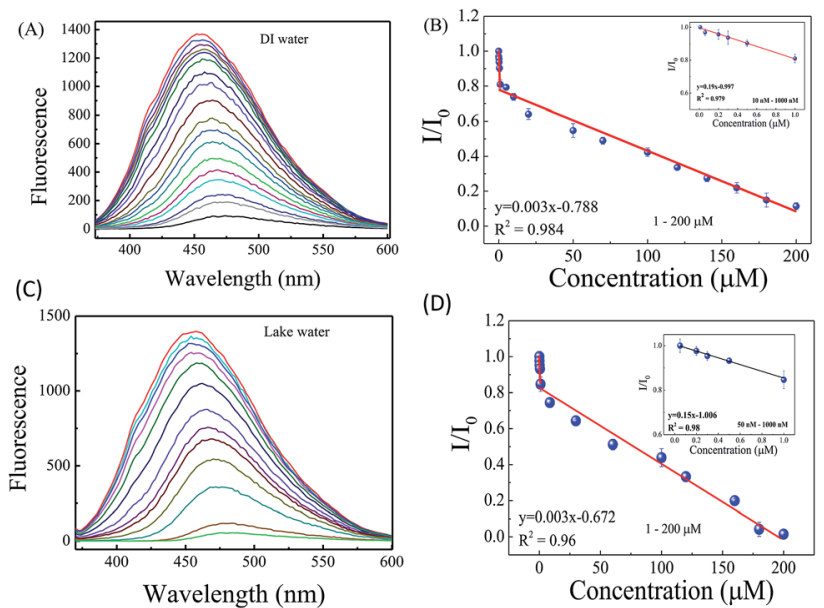

(D)

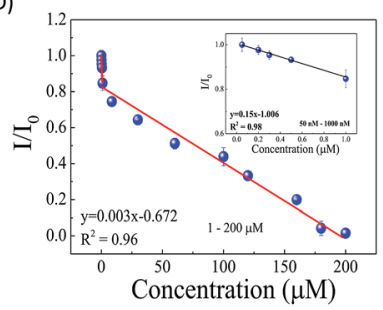

Fig. 5 The (A) fluorescence emission spectra of S-GQDs in the presence of 4-NP at $10 \mathrm{nM}-200 \mu \mathrm{M}$ in deionized water, (B) the calibration curve of 4-NP by S-GQDs in deionized water, the (C) fluorescence emission spectra of S-GQDs at 0.05-200 $\mu \mathrm{M} 4-\mathrm{NP}$ in lake water, and (D) the calibration curve of 4-NP by S-GQDs in lake water. The insets of Fig. 5B and $D$ are the calibration curves of low concentration of 4-NP in deionized and lake waters, respectively.

of various concentrations of $4-\mathrm{NP}$, the initial fluorescence intensity of S-GQDs in the absence of 4-NP $\left(F_{0}\right)$ is fixed in all the measurements. Therefore, the ratio of maximum peak intensity before and after the addition of 4-NP can be used to detect 4-NP. It is noteworthy that the as-prepared GQDs exhibit less fluorescence quenching effect in comparison with S-GQDs and only $29 \%$ decrease in fluorescence intensity are observed at $200 \mu \mathrm{M}$ 4-NP (Fig. S7, ESI $\dagger$ ), clearly showing that the doping with S atoms can enhance the analytical sensitivity toward 4-NP detection.

Fig. 5B shows the change in fluorescence intensity ratio $(F /$ $F_{0}$ ) as a function of 4-NP concentration where $F$ and $F_{0}$ are the fluorescence intensity of S-GQDs in the presence and absence of 4-NP, respectively. A two-stage linear relationship between the
$F / F_{0}$ and 4-NP concentration is obtained. The $F / F_{0}$ decreases rapidly in the 4-NP concentration range of 10-1000 nM, and then a linear decrease with a correlation coefficient $\left(r^{2}\right)$ of 0.984 is observed when the 4-NP concentration in the deionized water increases from 1 to $200 \mu \mathrm{M}$. Moreover, the low 4-NP concentration also exhibits a good linearity with $r^{2}$ of 0.979 (Inset of Fig. 5B). Several studies have indicated the 2-stage linear behavior when carbon-based quantum dots were used as the sensing elements. ${ }^{27,29,34,43}$ Tang et al. ${ }^{43}$ have fabricated $\mathrm{rGO} / \mathrm{Au}$ based nanosensor to detect 4-NP and a two-linear relationship in the concentration range of $0.05-2.0 \mu \mathrm{M}$ and $4.0-100 \mu \mathrm{M}$ was obtained. Ganganboina et al. ${ }^{29}$ have prepared the N-doped GQDdecorated $\mathrm{V}_{2} \mathrm{O}_{5}$ nanosheet for fluorescence detection of cysteine and a two-stage linear response to cysteine in the concentration range of 0.1-15 $\mu \mathrm{M}$ and 15-125 $\mu \mathrm{M}$ was observed because of the heterogeneously surface-mediated reaction. Our previous study ${ }^{34}$ has indicated that 4-NP can be rapidly adsorbed onto the Au@S-GQD surface by $\pi-\pi$ interaction at low 4-NP concentration and then slowly occupy the active sites on the Au@S-GQD surface at high concentration, resulting in the two different linear regions at low and high concentrations when UV-visible ratiometric method was used. In this study, we also found that 4 -NP can react with S-GQD by $\pi-\pi$ interaction, and subsequently quenches the fluorescence intensity of S-GQDs by a two-stage linear relationship. The LOD, determined by the $3 \sigma /$ $S$, where $\sigma$ is the standard deviation of the lowest signal and $S$ is the slope of linear calibration plot, is $0.7 \mathrm{nM}$, which is superior to the method using UV-visible ratiometric and other methods. $^{34,43}$ Since this phenomenon is mainly based on the physically surface-mediated reaction, one LOD value is sufficient to represent the sensitive of the S-GQD based sensing platform.

To further understand the applicability of the developed sensing platform on the detection of 4-NP in real water and wastewater environments, the contaminated lake water collected from NTHU campus was used as the model matrix. As shown in Fig. 5C, the fluorescence intensity of S-GQDs

Table 1 Analytical performance of 4-nitrophenol in aqueous solutions using different types of sensing probes

\begin{tabular}{|c|c|c|c|c|c|}
\hline Method & Probe & Sample matrix & Linear range $(\mu \mathrm{M})$ & $\mathrm{LOD}(\mathrm{nM})$ & Ref. \\
\hline \multirow[t]{3}{*}{ Electrochemistry } & $\mathrm{rGO} / \mathrm{Au}$ NPs & Lake water & $\begin{array}{l}0.05-2 \\
4-100\end{array}$ & 10 & 43 \\
\hline & $\mathrm{ZnO} / \mathrm{GCE}$ & DI water & $1-400$ & 20 & 6 \\
\hline & $\mathrm{rGO} / \mathrm{GCE}$ & Acetate buffer & $50-800$ & 42 & 44 \\
\hline \multirow[t]{4}{*}{ Fluorescence } & BSA Au-NCs & DI water & $0.001-0.5$ & 1 & 10 \\
\hline & MIP-C-dots & DI water & $0.2-50$ & 60 & 9 \\
\hline & QD@MIPs & DI water & $0.2-8$ & 51 & 12 \\
\hline & CdTe@MIP & DI water & $1-30$ & 40 & 11 \\
\hline \multirow[t]{2}{*}{ UV-visible } & Au@S-GQD & DI water & $\begin{array}{l}0.005-1 \\
1-50\end{array}$ & 3.5 & 34 \\
\hline & & Food wastewater & $\begin{array}{l}0.01-1.8 \\
1.8-50\end{array}$ & 8.4 & \\
\hline \multirow[t]{2}{*}{ Fluorescence } & S-GQDs & DI water & $\begin{array}{l}0.01-1.0 \\
1.0-200\end{array}$ & 0.7 & This study \\
\hline & & Lake water & $\begin{array}{l}0.05-1.0 \\
1.0-200\end{array}$ & 3.5 & \\
\hline
\end{tabular}


decreases with the increase in 4-NP concentration and around $95 \%$ decrease in fluorescence intensity is observed at $200 \mu \mathrm{M} 4$ NP in comparison with the pure S-GQDs in the absence of 4-NP. Similar to the sensing behavior in deionized water, the 4-NP detection by S-GQD in lake water exhibits good linear relationship in the concentration ranges of $0.05-1 \mu \mathrm{M}$ and $1-200 \mu \mathrm{M}$ with $r^{2}$ of 0.98 and 0.96 , respectively (Fig. 5D). In addition, the LOD of 4-NP detection in lake water is calculated to be $3.5 \mathrm{nM}$, which is higher than that in deionized water because of the high TOC concentration in lake water. It is also interesting to note that the fluorescence emission of S-GQDs is stable and the intensity remains unchanged after 6 months of storage in air at room temperature (Fig. S8, ESI $\dagger$ ). Although the two-stage linearity is obtained in wastewater, the use of the quantitative indicator, $F / F_{0}$, is still valid to indicate the suitable concentration range for practical application. These results clearly depict that the S-GQD is a stably excellent sensing probe, which can sensitively detect 4-NP in aqueous solutions with various matrices.

Table 1 shows the linear range and LOD value of 4 -NP detected by various optical- and electrochemical-based sensing nanomaterials. Several studies have used electroactive nanomaterials as the sensing element for the detection of 4-NP in different water matrices, and the dynamic range of $4-\mathrm{NP}$ is $2-3$ orders of magnitude with LOD values of 10-42 nM.,43,44 In addition, the fluorescence methods using Au nanocrystal as the fluorescence probe have been developed to detect 4-NP in deionized water and a linear range of $0.001-0.5 \mu \mathrm{M}$ with LOD of $1 \mathrm{nM}$ was observed. ${ }^{10}$ Moreover, the fluorescence sensors fabricated by MIP and quantum dots have been fabricated for the detection of 4-NP.9,11,12 The dynamic range of MIP-CD-based sensors is in the range of $0.2-50 \mu \mathrm{M}$ with LOD of 40-60 nM.,11,12 In this study, a wide dynamic range of 4 orders of magnitude with low LOD values of $0.7-3.5 \mathrm{nM}$ in different matrices of aqueous solution is obtained, which is superior to the most reported data shown in Table 1.

The high selectivity of sensing probe toward target compound detection is always important for the successful application to real samples. Therefore, the selectivity of S-GQD based sensing probe was further evaluated by adding 9 different aromatic compounds and nitroarenes into deionized water. Moreover, concentration of interferences used was $200 \mu \mathrm{M}$, which is 4 times higher than that of $4-\mathrm{NP}(50 \mu \mathrm{M})$. Fig. 6A shows the effect of interference species on the $F / F_{0}$ ratio of S-GQD in the presence of 4 -NP. It is clear that the $F / F_{0}$ ratio of S-GQDs in the presence of $50 \mu \mathrm{M} 4-\mathrm{NP}$ and most interference species including hexane, CNB, TNT, benzene, catechol, hydroquinone, and resorcinol remains almost unchanged $(<10 \%)$. Although addition of $200 \mu \mathrm{M} 2-\mathrm{NP}$ and phenol exhibits a relatively obvious decrease in $F / F_{0}$ ratio (62-76\%) in comparison with other interference species, the change in $F / F_{0}$ is still much lower than that of 4-NP. After mixing each interference with 4-NP in solution, the $F / F_{0}$ ratios of S-GQDs in all mixtures are in the range of $0.129-0.35$, which is almost the same as 4-NP only $\left(F / F_{0}\right.$ $=0.125$ ). This result clearly indicates the superior selectivity of S-GQDs toward 4-NP detection. The high selectivity of 4-NP is mainly attributed to the resonance stability and steric effect. It is noteworthy that $\mathrm{p} K_{\mathrm{a}}$ values of 4-NP and 2-NP are 6.90 and 7.2, respectively, ${ }^{45}$ which mean that nitrophenol would produce nitrophenolate ions at $\mathrm{pH}$ 7.0. The negatively charged $\mathrm{O}$ atoms on 4-nitrophenolate ions can be delocalized throughout the benzene ring and become more resonance-stabilized than those of 2-nitrophenolate ions and other aromatic compounds. ${ }^{45,46}$ In addition, the steric hindrance effect lowers the inductive effect of the nitro group on 2-nitrophenolate ions compared with that of 4-nitrophenolate ions. Therefore, S-GQDs have a high selectivity on 4-NP detection in comparison with other aromatic compounds selected in this study.

The quenching mechanism for 4-NP detection by S-GQDs can be explained by both dynamic quenching and static quenching. Fig. 6B shows the time-resolved fluorescence spectra of S-GQDs in the absence and presence of 50-200 $\mu \mathrm{M} 4$ NP. The fluorescence decay of S-GQDs in the absence of 4-NP is $7.1 \mathrm{~ns}$ and becomes more rapid after the addition of 4-NP. The fluorescence emission is found to decay on a time scale of 7.09 ns at $50 \mu \mathrm{M} 4$-NP and then again decreases to $7.01 \mathrm{~ns}$ when the concentration of 4 -NP increases to $200 \mu \mathrm{M}$, showing that the fluorescence quenching mechanism is a dynamic quenching process. The significant quenching may occur via the $\pi-\pi$ interaction and hydrogen bonding between 4-NP and S-GQDs. The primary $\pi$-bond networks on the surface of S-GQDs play the crucial role in recognizing the target compound without
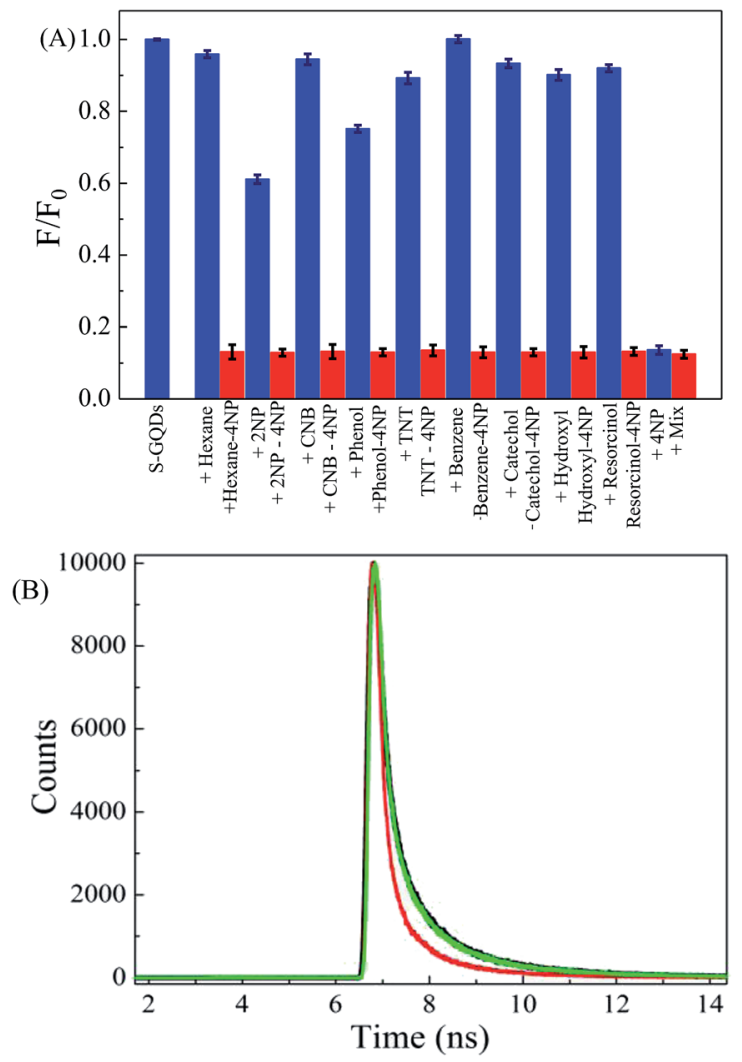

Fig. 6 (A) Competitive selectivity of S-GQDs towards $50 \mu \mathrm{M} 4-N P$ in the presence of $200 \mu \mathrm{M}$ other interfering reagents and (B) timeresolved fluorescence spectra of S-GQDs in the presence of various concentrations of 4-NP ranging from 0-200 $\mu \mathrm{M}$ (black line: $0 \mu \mathrm{M}$; green line: $50 \mu \mathrm{M}$; red line: $200 \mu \mathrm{M}$ ). 
Table 2 Recovery of various 4-NP concentrations in deionized water ranging from $10 \mathrm{nM}$ to $50 \mu \mathrm{M}$ using S-GQDs as the fluorescence probe

\begin{tabular}{lllr}
\hline Methods & Added concentration & Detected concentration & $\begin{array}{l}\text { Recovery }(\%) \\
(n=3)\end{array}$ \\
\hline S-GQD method & $10 \mathrm{nM}$ & $11 \mathrm{nM}$ & $110 \pm 2$ \\
Traditional UV-visible method & $10 \mu \mathrm{M}$ & $10.3 \mu \mathrm{M}$ & $103 \pm 7$ \\
& $50 \mu \mathrm{M}$ & $48.9 \mu \mathrm{M}$ & $98 \pm 5$
\end{tabular}

treatment and passivation. Consequently, the fluorescence emission of S-GQDs is slightly red-shifted after addition of high concentration of 4-NP.

\section{Detection of 4-NP in wastewater}

To evaluate the reliability and applicability of the developed method, the fluorescence S-GQDs probe was further used for the detection of 4-NP in different real wastewater samples by standard addition method. Moreover, the recovery of 4-NP in deionized water using S-GQD sensing probe and traditional UVvisible method was examined and compared. As illustrated in Table 2, the recovery of low concentration of $10 \mathrm{nM} 4$-NP by SGQDs is $110 \pm 2 \%$ in deionized water. Different from the $S$ GQD sensing probe, the traditional UV-visible method can only detect 4-NP $>1 \mu \mathrm{M}$, and the recoveries of 10 and $50 \mu \mathrm{M}$ of 4 NP by UV-visible method are $103 \pm 7 \%$ and $98 \pm 5 \%$, respectively. It is noteworthy that the fluorescence S-GQD probe can detect low concentration of 4-NP and the recovery is comparable with the traditional UV-visible method, clearly indicating the superiority of S-GQD as the sensing element for 4-NP detection.

The real sample study was conducted by selecting three wastewater samples with different matrices including the industrial wastewater treatment plants and contaminated lake water. Table 3 shows the analytical performance of 4-NP using SGQDs as the fluorescence probe in wastewaters. A total of 36 samples in 4 different categories were analyzed. The 4-NP concentration in all wastewaters are lower than the LOD value, and, therefore, known concentrations of 4-NP were spiked into the wastewater to understand the matrix effect of real samples.
The spiked concentrations of 4-NP were in the range of $100 \mathrm{nM}-$ $100 \mu \mathrm{M}$ to cover all the possible contaminated ranges in wastewater. After spiking medium (0.1-1 $\mu \mathrm{M})$ and high (10-100 $\mu \mathrm{M})$ concentrations of $4-\mathrm{NP}$ into wastewater, the detected concentrations of 4-NP are close to the spiked ones and the detected percentages of spiked 4-NP are in the range of (98 \pm 4$)-$ $(108 \pm 2) \%$, clearly depicting that S-GQD is a promising fluorescence probe to effectively monitor a wide range of 4-NP concentration in real wastewater.

\section{Rapid screening of 4-NP using S-GQD paper based sensors}

The simplicity of sensing probe is also one of the important parameters to evaluate the applicability of nanosensors. After successful application of S-GQDs to selectively and sensitively detect 4-NP in different matrices of wastewaters, the S-GQDbased paper sensor was fabricated to rapidly screen 4-NP in wastewater according to our previous report. ${ }^{24}$ The wastewater sample was chosen from metal industry. Fig. 7a illustrates the optical image of S-GQD-based paper sensor after immersing into wastewater spiking with $0.1-500 \mu \mathrm{M} 4-\mathrm{NP}$ for $1 \mathrm{~min}$. In the absence of 4-NP, the paper sensor emits a bright blue fluorescence after the irradiation of $330 \mathrm{~nm}$ UV light. In contrast, the fluorescence of S-GQD paper strip turns into faint upon the increase in 4-NP concentration in wastewater, clearly indicating that the developed S-GQD paper strip can serve as a sensing platform for the rapid screening of a wide concentration range of 4-NP in wastewater. Besides, the paper strip based sensing platform shows excellent selectivity toward 4-NP detection. As shown in Fig. 7b, the color of paper strip based sensing probe in

Table 3 Analytical performance of using S-GQDs as the fluorescence probe for the detection of various 4-NP concentrations in different wastewaters ranging from 0.05 to $100 \mu \mathrm{M}$

\begin{tabular}{|c|c|c|c|}
\hline Sample name & Added concentration & Detected concentration & $\begin{array}{l}\text { Detected } \\
(n=3)\end{array}$ \\
\hline \multirow[t]{3}{*}{ Metal industrial wastewater } & $50 \mathrm{nM}$ & $49 \mathrm{nM}$ & $98 \pm 4$ \\
\hline & $100 \mathrm{nM}$ & $99 \mathrm{nM}$ & $99 \pm 3$ \\
\hline & $1 \mu \mathrm{M}$ & $1.07 \mu \mathrm{M}$ & $107 \pm 5$ \\
\hline \multirow[t]{3}{*}{ Electroplating wastewater } & $100 \mathrm{nM}$ & $105 \mathrm{nM}$ & $105 \pm 4$ \\
\hline & $500 \mathrm{nM}$ & $490 \mathrm{nM}$ & $98 \pm 5$ \\
\hline & $10 \mu \mathrm{M}$ & $10.2 \mu \mathrm{M}$ & $102 \pm 5$ \\
\hline \multirow[t]{3}{*}{ Sewage } & $200 \mathrm{nM}$ & $199 \mathrm{nM}$ & $99.5 \pm 2$ \\
\hline & $20 \mu \mathrm{M}$ & $21 \mu \mathrm{M}$ & $105 \pm 3$ \\
\hline & $50 \mu \mathrm{M}$ & $51.5 \mu \mathrm{M}$ & $103 \pm 5$ \\
\hline \multirow[t]{3}{*}{ Lake water } & $1 \mu \mathrm{M}$ & $1.03 \mu \mathrm{M}$ & $103 \pm 3$ \\
\hline & $10 \mu \mathrm{M}$ & $10.8 \mu \mathrm{M}$ & $108 \pm 2$ \\
\hline & $100 \mu \mathrm{M}$ & $102 \mu \mathrm{M}$ & $102 \pm 6$ \\
\hline
\end{tabular}



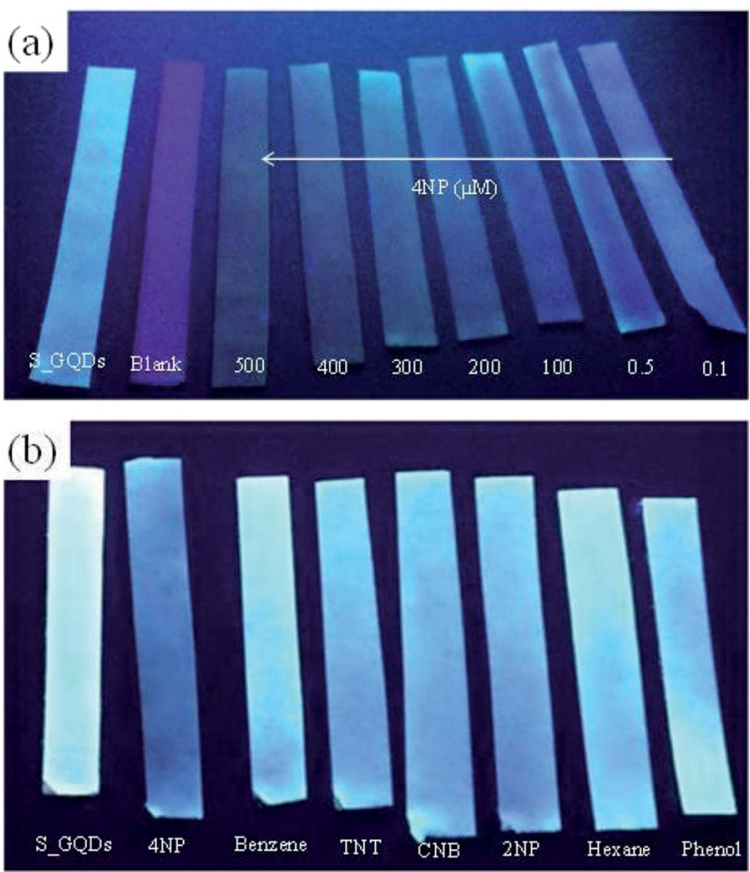

Fig. 7 (a) The change in the fluorescence intensity as a function of 4$\mathrm{NP}$ concentrations ranging from $0.1-500 \mu \mathrm{M}$ and effect of (b) $200 \mu \mathrm{M}$ organic interferences including benzene, TNT, CNB, 2-NP, hexane and phenol on 4-NP detection.

the presence of $200 \mu \mathrm{M}$ interferences including hexane, phenol, TNT, benzene, 2-NP and CNB is similar to that of blank. However, the color of S-GQDs based paper strip changes to blue when $0.5 \mu \mathrm{M} 4-\mathrm{NP}$ is added to the wastewater, clearly depicting the high flexibility of using S-GQD paper-based sensors to rapid detection of 4-NP in real wastewater effluents. The preparation of environmentally benign GQD-based nanomaterials as well the increase in quantum yield is always important for environmental application. In this study, the quantum yield still leaves a room to improve. It is noteworthy that agricultural products such as orange, grape and citron contain plenty of organic constituents like glucose and citric acid as well as small amounts of nitrogen and sulfur, which are ideal raw materials for the preparation of environmentally friendly doped-GQDs. Further study on the fabrication of doped-GQDs with high quantum yield using agricultural products as the starting materials would be a green and novel synthesis route for the preparation of low cost and environmental benign sensing materials for environmental application in water and wastewater.

\section{Conclusions}

In this study, we have developed a novel and simple synthesis method for the highly sensitive and selective detection of 4-NP in water and wastewater samples with different matrices. The uniformly distributed S-GQDs with diameter of 1-5 $\mathrm{nm}$ can stably emit fluorescence at $450 \mathrm{~nm}$ to act as the sensing element, and the doping with $\mathrm{S}$ atoms enhances the fluorescence quantum yield. 4-Nitrophenol can serves as the fluorescence quencher to extinct the fluorescence intensity by $\pi-\pi$ interaction with the graphitic carbon backbone of GQDs, thus making S-GQD a promising sensing element for highly sensitive detection of 4-NP. The S-GQDs display an excellent ability toward 4-NP detection and a dynamic range of 3-4 orders of magnitude with LOD value of $0.7 \mathrm{nM}$ in deionized water and $3.5 \mathrm{nM}$ in wastewater is obtained. Moreover, the S-GQD based sensing platform can be employed for the determination of 4$\mathrm{NP}$ in different matrices of water and wastewater and the detected percentages of $(98 \pm 4)-(108 \pm 2) \%$ are obtained at 4 NP concentration of $0.05-100 \mu \mathrm{M}$. Furthermore, S-GQDs paper base sensors demonstrates the applicability to rapid screen of 0.1-500 $\mu \mathrm{M}$ 4-NP in wastewater without obvious matrix interference. In conclusion, our results clearly indicate the superiority of S-doped GQDs to rapidly and efficiently detect 4-NP, which can pave a new way to fabricate GQD-based fluorescence probe to monitor nitroarenes and other contaminants in aqueous solution with a wide variety of matrix effects.

\section{Conflicts of interest}

There are no conflicts to declare.

\section{Acknowledgements}

The authors thank the Ministry of Science and Technology (MOST), Taiwan for financial support under grant no. MOST 105-2113-M-009-023-MY3 and 107-2911-I-007-302.

\section{References}

1 V. Inglezakis, S. Malamis, A. Omirkhan, J. Nauruzbayeva, Z. Makhtayeva, T. Seidakhmetov and A. Kudarova, J. Environ. Manage., 2017, 203, 825-830.

2 I. Tapsoba, S. Bourhis, T. Feng and M. Pontie, Electroanalysis, 2009, 21, 1167-1176.

3 F. H. Lin and R. A. Doong, J. Phys. Chem. C, 2017, 121, 78447853.

4 F. H. Lin and R. A. Doong, J. Phys. Chem. C, 2017, 121, 78447853.

5 J. J. Lv, A. J. Wang, X. H. Ma, R. Y. Xiang, J. R. Chen and J. J. Feng, J. Mater. Chem. A, 2015, 3, 290-296.

6 A. Santhoshkumar, H. P. Kavitha, R. Suresh, J. P. Venila, S. P. Kumar and V. Narayanan, J. Mater. Sci.: Mater. Electron., 2017, 28, 9272-9278.

7 Y. Q. Cheng, Y. H. Li, D. Li, B. Zhang, R. F. Hao and S. B. Sang, Int. J. Electrochem. Sci., 2017, 12, 7754-7764.

8 A. E. Vilian, S. R. Choe, K. Giribabu, S. C. Jang, C. Roh, Y. S. Huh and Y. K. Han, J. Hazard. Mater., 2017, 333, 54-62.

9 S. Wu, S. Fan, S. Tan, J. Wang and C. P. Li, RSC Adv., 2018, 8, 775-784.

10 X. Yang, J. Wang, D. Su, Q. Xia, F. Chai, C. Wang and F. Qu, Dalton Trans., 2014, 43, 10057-10063.

11 T. Hao, X. Wei, Y. Nie, Y. Xu, Y. Yan and Z. Zhou, Microchim. Acta, 2016, 183, 2197-2203. 
12 J. Yu, X. Wang, Q. Kang, J. Li, D. Shen and L. Chen, Environ. Sci.: Nano, 2017, 4, 493-502.

13 M. Hassan, E. Haque, K. R. Reddy, A. I. Minett, J. Chen and V. G. Gomes, Nanoscale, 2014, 6, 11988-11994.

14 Y. Yan, Q. Liu, X. Du, J. Qian, H. Mao and K. Wang, Anal. Chim. Acta, 2015, 853, 258-264.

15 A. B. Ganganboina, A. Dutta Chowdhury and R. A. Doong, ACS Sustainable Chem. Eng., 2017, 5, 4930-4940.

16 S. Zhu, X. Yan, J. Sun, X.-E. Zhao and X. Wang, Talanta, 2019, 200, 163-168.

17 X. Yan, X. E. Zhao, J. Sun, S. Zhu, C. Lei, R. Li, P. Gong, B. Ling, R. Wang and H. Wang, Sens. Actuators, B, 2018, 263, 27-35.

18 X. E. Zhao, C. Lei, Y. Gao, H. Gao, S. Zhu, X. Yang, J. You and H. Wang, Sens. Actuators, B, 2017, 253, 239-246.

19 X. Zhao, C. Lei, Y. Wang, F. Qu, S. Zhu, H. Wang and J. M. You, RSC Adv., 2016, 6, 72670-72675.

20 A. Ananthanarayanan, X. Wang, P. Routh, B. Sana, S. Lim, D. H. Kim, K. H. Lim, J. Li and P. Chen, Adv. Funct. Mater., 2014, 24, 3021-3026.

21 J. Lu, M. Yan, L. Ge, S. Ge, S. Wang, J. Yan and J. Yu, Biosens. Bioelectron., 2013, 47, 271-277.

22 F. Wang, Z. Gu, W. Lei, W. Wang, X. Xia and Q. Hao, Sens. Actuators, B, 2014, 190, 516-522.

23 A. Dutta Chowdhury and R. A. Doong, ACS Appl. Mater. Interfaces, 2016, 8, 21002-21010.

24 N. T. N. Anh, A. D. Chowdhury and R. A. Doong, Sens. Actuators, B, 2017, 252, 1169-1178.

25 K. Bindumadhavan, P. Y. Chang and R. A. Doong, Electrochim. Acta, 2017, 243, 282-290.

26 X. Li, S. P. Lau, L. Tang, R. Ji and P. Yang, Nanoscale, 2014, 6, 5323-5328.

27 A. Ananthanarayanan, Y. Wang, P. Routh, M. A. Sk, A. Than, M. Lin, J. Zhang, J. Chen, H. Sun and P. Chen, Nanoscale, 2015, 7, 8159-8165.

28 Z. Yan, X. Qu, Q. Niu, C. Tian, C. Fan and B. Ye, Anal. Methods, 2016, 8, 1565-1571.
29 A. B. Ganganboina, A. Dutta Chowdhury and R. A. Doong, ACS Appl. Mater. Interfaces, 2017, 10, 614-624.

30 X. Wang, G. Sun, P. Routh, D. H. Kim, W. Huang and P. Chen, Chem. Soc. Rev., 2014, 43, 7067-7098.

31 S. Li, Y. Li, J. Cao, J. Zhu, L. Fan and X. Li, Anal. Chem., 2014, 86, 10201-10207.

32 S. Bian, C. Shen, H. Hua, L. Zhou, H. Zhu, F. Xi, J. Liu and X. Dong, RSC Adv., 2016, 6, 69977-69983.

33 S. Bian, C. Shen, Y. Qian, J. Liu, F. Xi and X. Dong, Sens. Actuators, B, 2017, 242, 231-237.

34 N. T. N. Anh and R. A. Doong, ACS Appl. Nano Mater., 2018, 1, 2153-2163.

35 A. B. Ganganboina, A. Dutta Chowdhury and R. A. Doong, ACS Appl. Mater. Interfaces, 2017, 10, 614-624.

36 A. E. Segneanu, I. Gozescu, A. Dabici, P. Sfirloaga and Z. Szabadai, Macro Nano Spectrosc., 2012, 145-164.

37 T. Alizadeh and M. Shokri, Sens. Actuators, B, 2016, 222, 728734.

38 J. C. Vinci, I. M. Ferrer, N. W. Guterry, V. M. Colon, J. F. Destino, F. V. Bright and L. A. Colon, Appl. Spectrosc., 2015, 69, 1082-1090.

39 M. Gholinejad, J. Ahmadi, C. Nájera, M. Seyedhamzeh, F. Zareh and M. K. Zareh, ChemCatChem, 2017, 9, 1442-1449.

40 X. Yang, M. Liu, Y. Yin, F. Tang, H. Xu and X. Liao, Sensors, 2018, 18, 964.

41 G. Wang, Q. Guo, D. Chen, Z. Liu, X. Zheng, A. Xu, S. Yang and G. Ding, ACS Appl. Mater. Interfaces, 2018, 10, 57505759.

42 N. Qin, Y. Liu, W. Wu, L. Shen, X. Chen, Z. Li and L. Wu, Langmuir, 2015, 31, 1203-1209.

43 Y. Tang, R. Huang, C. Liu, S. Yang, Z. Lu and S. Luo, Anal. Methods, 2013, 5, 5508-5514.

44 P. Wiench, B. Grzyb, Z. Gonzalez, R. Menendez, B. Handke and G. Gryglewicz, J. Electroanal. Chem., 2017, 787, 80-87.

45 T. B. Nguyen, C. P. Huang and R. A. Doong, Appl. Catal., B, 2019, 240, 337-347.

46 F. H. Lin and R. A. Doong, J. Phys. Chem. C, 2017, 121, 78447853. 